\title{
EDITORIAL
}

\section{CHRONIC NON COMMUNICABLE DISEASES AND ITS RISK FACTORS IN BANGLADESH}

MD. MUJIBUR RAHMAN

In recent years globalization and technological advances have combined to create a potent force for a reduction in deaths from communicable diseases not only in the industrialized nation but also in the developing countries. As a result there is an increase in average life expectancy, and an epidemic rise in chronic non-communicable diseases (NCDs) such as heart disease, stroke, cancer, and chronic obstructive pulmonary disease (COPD). ${ }^{1}$ The term chronic noncommunicable disease is used to refer to major chronic disorders such as heart disease, stroke, cancer, and COPD. These diseases share common, modifiable risk factors, in the causal pathway. ${ }^{2}$ Mental disorders (other than stroke) and chronic illnesses related to infectious processes (such as hepatitis B or human papilloma virus infection) are not covered in the usual description of NCDs.

NCDs were estimated to have contributed to almost $60 \%$ of deaths globally and among them about $80 \%$ occur in the developing countries. ${ }^{3}$ NCDs are already of major importance in developed countries and are rapidly becoming a major public health threat in the developing world. These diseases constituted $43 \%$ of the global burden of disease in 1999. Based on current trends, by 2020 they will account for $73 \%$ of deaths and $60 \%$ of the disease burden in the developing countries. ${ }^{4}$

Bangladesh is now facing a double burden of diseases contributing both from communicable and non communicable diseases. The underlying cause of NCDs epidemic is the increase in lifestyle related risk factors resulting from social and economic changes. In many countries the increasing impact of globalization has given momentum to this process. ${ }^{2}$ Currently neighbouring India is also experiencing an epidemic of NCDs attributed to lifestyle changes resulting from urbanization. ${ }^{5}$

\section{Disability and death}

In terms of disability, chronic non-communicable disease was estimated to account for $61 \%$ of total disability-adjusted life years in people aged 15-59 years, and $84 \%$ of the burden in those aged 60 years and older in 2008. The burden is expected to rise to $74 \%$ and $89 \%$, respectively, by the year $2030 .{ }^{4}$ These estimates were based on the assumption that risk factor exposures, particularly tobacco use and being overweight or obese, will fall with economic development and improving health systems in countries with low and middle incomes. Should this assumption not be met, the future burden from chronic non-communicable diseases is expected to be larger than has been predicted.

Death and disability from chronic non-communicable diseases can exert an economic burden in two waysindirectly, through loss of productivity and income, and directly, through household spending on chronic medical care, often of catastrophic proportions. ${ }^{6}$ This situation has serious consequences not only at the economic growth of the nation but also at the individual family level.

\section{Risk factors}

The chronic non communicable diseases have got some common risk factors which are environmental risk factors like globalization, urbanization, poverty, low education and stress. All these risk factors lead to the development of behavioral risk factors like tobacco use, unhealthy diet and physical inactivity. The behavioral risk factors are being responsible for the development of biological risk factors like raised $\mathrm{BP}$, diabetes mellitus, abnormal blood lipids, increased waist circumference and abnormal lung functions. A small number of risk factors account for a large proportion of the global burden of chronic non communicable diseases. ${ }^{8}$ Data from the Asia- Pacific Cohort Studies Collaboration suggest that these risk factors have the same effects in Asian populations as in others. ${ }^{9}$

To respond to the increased burden of NCDs, WHO put due emphasis on gathering information on NCDs and their risk factors, as they are necessary for designing prevention and control programs with specific goal and measurable outcomes. Population specific data on risk factors are essential in-order to set priorities, develop targeted program on NCDs.

Professor, Department of Medicine, Shaheed Suhrawardy Medical College, Dhaka.

Bangladesh J Medicine 2012; 23 : 48-49 
Anticipating the NCD epidemic, WHO has initiated the worldwide STEPwise approach to Surveillance (STEPS) of risk factors for NCDs. STEPS focuses on the periodic collection of data on key risk factors associated with major NCDs which is indispensable for designing community based interventions to reduce risk factors in the population. ${ }^{10}$ Prevalence of common NCDs are increasing and most of these NCDs share common preventable risk factors. Major risk factors of today will be the diseases of tomorrow. Therefore measuring risk factors for NCDs is an attempt to predict the future distribution of NCDs in a population and is vital for promoting disease prevention and control programmes. ${ }^{11}$

\section{Bangladesh Situation}

Bangladesh Society of Medicine in collaboration with and support from WHO has conducted a nationwide survey on the NCDs risk factors in Bangladesh. This is the first ever survey of this kind representing the whole population in our country which provides essential information on key indicators of NCD risk factors and creates an opportunity for policy makers, program managers, and researchers to adopt interventions. Inadequate intake of fruit and vegetables, use of tobacco, low level of physical activity (especially in women), binge drinking among current drinkers, obesity (especially abdominal), high blood pressure and diabetes mellitus are fairly common in adults. Tobacco was being used by half of the adult population (51\%), man being the most users $(70 \%)$ compared to women (34.4\%). About $96 \%$ of the population did not consume adequate fruit or vegetables on an average day. The prevalence of low physical activity among the surveyed population was $27 \%$ with highest distribution among the females (41.3\%). The prevalence of hypertension was $17.9 \%$. Almost all adults (98.7\%) have at least one risk factor and substantial proportion of people have two or more risk factors. ${ }^{12}$ The 2010 survey did not include the measurement of blood sugar and the lipid abnormalities. So this is the high time to do the step 3 of the STEPS survey.

Bangladesh is having a burden of both communicable and non communicable diseases. We have to undertake adequate measures to control and prevent the non communicable diseases together with communicable diseases. The Government of Bangladesh has taken right steps in translating the evidence relating to NCDs and using those data for prevention and control programs for the nation. The NCD unit of Directorate General of Health Services has taken a Strategic Plan for Surveillance and
Prevention of NCDs with inputs generated through an extensive process within the various domains of NCDs and is reflective of broad based consensus. It puts emphasis on the strengths of partnerships and outlines a scope of interventions that are built on shared responsibility of various sectors of the Government according to their own missions and strategies.

\section{Reference:}

1. WHO. The global burden of disease: 2004 update. Geneva, Switzerland: World Health Organization, 2008.

2. WHO. Preventing chronic diseases-a vital investment. Geneva, Switzerland: World Health Organization, 2005.

3. World Health Organization. Global status report on non communicable diseases 2010. WHO, Geneva 2011.

4. World Health Organization. WHO NCD Surveillance Strategy. Available at Available at <http:// www.who.int/ncd_surveillance/strategy/en / print.html>. Accessed on 24 march 2013.

5. WHO. Preventing chronic diseases-a vital investment. Geneva, Switzerland: World Health Organization, 2005.

6. Reddy KS, Shah B, Varghese C, Ramadoss A. Responding to the threat of chronic diseases in India. Lancet 2005; 366:1744-49.

7. Sun Q, Liu X, Meng Q, Tang S, Yu B, Tolhurst R. Evaluating the fi nancial protection of patients with chronic disease by health insurance in rural China. Int J Equity Health 2009; 8: 42.

8. Antonio Dans, Nawi Ng, Cherian Varghese, E Shyong Tai, Rebecca Firestone, Ruth Bonita The rise of chronic non-communicable diseases in Southeast Asia: time for action. Lancet 2011; 337: 680-89

9. Yusuf S, Hawken S, Ounpuu S, et al, on behalf of the INTERHEART Study Investigators. Effect of potentially modifiable risk factors associated with myocardial infarction in 52 countries (the INTERHEART study): case-control study. Lancet 2004; 364: 937-52.

10. Asia Pacific Cohort Studies Collaboration. Cholesterol, diabetes and major cardiovascular diseases in the AsiaPacific region. Diabetologia 2007; 50: 2289-97.

11. Bonita R, de Courten M, Dwyer T, Jamrozik K, Winkelmann R. Surveillance of risk factors for noncommunicable diseases: The WHO STEPwise approach summary. WHO Geneva 2001

12. Magnus $\mathrm{P}$, Beaglehole R. The real contribution of the major risk factors to the coronary epidemics: time to end the "only-50\%" myth. Arch Intern Med 2001; 161: 257-60.

13. WHO. Non-communicable disease risk factor survey, Bangladesh 2010. WHO Dhaka, 2010. 